\title{
Procalcitonin-guided antibiotic therapy in intensive care unit patients: a systematic review and meta-analysis
}

\author{
Hui-Bin Huang ${ }^{1,2}$, Jin-Min Peng ${ }^{1}$, Li Weng ${ }^{1}$, Chun-Yao Wang ${ }^{1}$, Wei Jiang ${ }^{1}$ and Bin Du*
}

\begin{abstract}
Background: Serum procalcitonin (PCT) concentration is used to guide antibiotic decisions in choice, timing, and duration of anti-infection therapy to avoid antibiotic overuse. Thus, we performed a systematic review and meta-analysis to seek evidence of different PCT-guided antimicrobial strategies for critically ill patients in terms of predefined clinical outcomes.

Methods: We searched for relevant studies in PubMed, Embase, Web of Knowledge, and the Cochrane Library up to 25 February 2017. Randomized controlled trials (RCTs) were included if they reported data on any of the predefined outcomes in adult ICU patients managed with a PCT-guided algorithm or according to standard care. Results were expressed as risk ratio (RR) or mean difference (MD) with accompanying 95\% confidence interval (CI).

Data synthesis: We included 13 trials enrolling 5136 patients. These studies used PCT in three clinical strategies: initiation, discontinuation, or combination of antibiotic initiation and discontinuation strategies. Pooled analysis showed a PCT-guided antibiotic discontinuation strategy had fewer total days with antibiotics (MD -1.66 days; $95 \% \mathrm{Cl}-2.36$ to -0.96 days), longer antibiotic-free days (MD 2.26 days; $95 \%$ Cl 1.40-3.12 days), and lower short-term mortality (RR $0.87 ; 95 \%$ Cl 0.76-0.98), without adversely affecting other outcomes. Only few studies reported data on other PCTguided strategies for antibiotic therapies, and the pooled results showed no benefit in the predefined outcomes.
\end{abstract}

Conclusions: Our meta-analysis produced evidence that among all the PCT-based strategies, only using PCT for antibiotic discontinuation can reduce both antibiotic exposure and short-term mortality in a critical care setting.

Keywords: Procalcitonin, Antibiotic strategies, Meta-analysis, Systematic review, Intensive care unit

\section{Background}

Timely diagnosis and appropriate antimicrobial treatment of infection remain a major challenge in critical care settings. Delay in diagnosis due to lack of specific clinical signs in the early stage of infection may withhold or delay antibiotic therapy. On the other hand, concern of not treating potentially life-threatening infection and the risk of recurrence frequently leads clinicians to antimicrobial overuse in intensive care unit (ICU) [1,2]. Studies have demonstrated that up to $50 \%$ of antibiotics

\footnotetext{
*Correspondence: dubin98@gmail.com

${ }^{1}$ Medical ICU, Peking Union Medical College Hospital, Peking Union Medical College and Chinese Academy of Medical Sciences, 1 Shuai Fu Yuan, Beijing 100730, People's Republic of China

Full list of author information is available at the end of the article
}

prescribed in hospital settings are either unnecessary or inappropriate [3]. Nowadays, long-term antimicrobial regimens applied to critically ill patients are common and often based on empiric rules $[4,5]$. This may result in increased medical costs, emergence of resistant pathogens, prolonged length of stay (LOS), and risk of mortality $[6,7]$.

Recently, procalcitonin (PCT) has shown to be a promising biomarker for identification of bacterial infections and is correlated with the severity of infection [8-11]. The 2016 Surviving Sepsis Campaign (SSC) guidelines offered a weak recommendation (low quality of evidence), favouring that measurement of procalcitonin levels can be used to support shortening the duration of antimicrobial therapy in sepsis patients [12]. However, 
one recent large study failed to show any benefit of daily PCT measurement with regard to time to appropriate therapy or survival, but resulted in a longer antibiotic course and ICU stay [13]. To date, several meta-analyses have assessed the value of PCT to guide antibiotic stewardship in ICU patients [14-22]. Findings of these reports showed that utilizing PCT to guide antibiotic decisions could significantly reduce antibiotics use, but did not improve patient outcomes, such as mortality, hospital, or ICU LOS. However, one major limitation of these metaanalyses was unexplainable significant heterogeneity among included trials, possibly due to the fact that the different PCT guidance strategies (including antibiotic initiation, discontinuation, or combination of antibiotic initiation and discontinuation strategies) had been evaluated in these trials. Since the considerable differences in methodologies and research purposes associated with the different PCT-guided strategies, the previous metaanalyses might not accurately evaluate the effects of PCTbased algorithms (Additional file 1: Table S1). Recently, two large-scale randomized controlled trials (RCTs) of PCT-guided antibiotic strategy in ICU patients have been published, with inconsistent results [23, 24]. Of note, the study by de Jong et al., the largest PCT trial to date, demonstrated an unexpected and significant survival benefit, in addition to less antibiotic exposure.

Therefore, with the aid of increased power of metaanalytic techniques, we sought to expand the previous analyses by including studies published recently, stratifying different strategies to have a more accurate analysis of the influence of different PCT algorithms to guide antimicrobial decisions.

\section{Methods}

\section{Search strategy and selection criteria}

This systematic review and meta-analysis were conducted in accordance with the PRISMA guidance [25]. We searched RCTs in PubMed, Embase, Web of Science, and Cochrane Central Register of Controlled Trials from inception through 25 February 2017 to identify potentially relevant studies. Search included the following key words: ("Procalcitonin" OR "PCT") AND ("intensive care" OR "critically ill" OR "critical care"). No language restriction was imposed. Reference lists of relative articles were also reviewed.

Studies were included if they are enrolling adult ICU patients, with confirmed or suspected infection, assigned to either a PCT-guided therapeutic strategy group or a standard care group. Standard care referred to antimicrobial regimens based on clinical signs, laboratory results, and empiric rules or guidelines, without consideration of PCT level. We excluded studies enrolling children or patients without any evidence of infection and studies without mentioning of PCT assay methods. Articles available only in abstract form or meeting reports were also excluded.

\section{Data extraction and outcomes}

Two reviewers independently extracted data from included studies on the first author, year of publication, country, sample size, study design, ICU type, compared protocols, methods of PCT assay, methodological quality, as well as all outcomes of interest.

We stratified different PCT-guided strategies according to medical decision with regards to antimicrobial therapy. In brief, strategy of antibiotic initiation referred to the decision to or not to start antibiotics, and decision of the intensified monitoring, diagnostic efforts, and interventions to explore uncontrolled sources of infection based on a predefined threshold of baseline PCT concentration, while strategy of antibiotic discontinuation meant making the decision to stop antibiotics according to a predefined threshold of PCT concentration, or the PCT level dropped by a certain proportion predefined compared with the previous value. The primary outcomes were the duration of antibiotic use and the short-term mortality, while the latter was defined as ICU or hospital or 28-day mortality [26, 27]. Secondary outcomes included ICU and hospital LOS.

\section{Quality assessment}

Two independent reviewers evaluated the quality of studies using the risk of bias tool recommended by the Cochrane Collaboration [28]. We assigned a value of high, unclear, or low to the following items: sequence generation; allocation concealment; blinding; incomplete outcome data; selective outcome reporting; and other sources of bias. Discrepancies were identified and resolved through discussion.

\section{Statistical analysis}

The results from all relevant studies were combined to estimate the pooled risk ratio (RR) and associated 95\% confidence intervals (CIs) for dichotomous outcomes. As to the continuous outcomes, mean differences (MD) and 95\% CI were estimated as the effect results. Heterogeneity was tested by using the $I^{2}$ statistic. An $I^{2}<50 \%$ was considered to indicate insignificant heterogeneity, and a fixed-effect model was used, whereas a random-effect model was used in cases of significant heterogeneity $\left(I^{2}>50 \%\right)$. Before data analysis, we estimated mean from median and standard deviations (SD) from IQR using the methods described in previous studies [29]. Sensitivity analyses were performed by excluding trials that potentially biased the results of primary outcomes. Publication bias was evaluated by visually inspecting funnel plots. All 
analyses were performed using Review Manager version 5.3 .

\section{Results \\ Study selection}

The literature search yielded 881 records through database searching, and 13 RCTs fulfilled inclusion criteria were eligible for the final analysis [13, 23, 24, 30-39]. The overview of the study selection process is presented in Fig. 1. In the study by Jensen et al., some patients without infection were also included [13]; therefore, we only included patients with severe sepsis or septic shock that fulfilled our inclusion criteria from this study. The Cochrane risk of bias score for each citation varied across the studies (Additional file 2: Table S2).

\section{Study characteristics}

The main characteristics and predefined outcomes of the 13 included studies are shown in Table 1 and Additional file 3: Table S3. The degree of non-compliance with PCT algorithm recommendations for antibiotics varied among

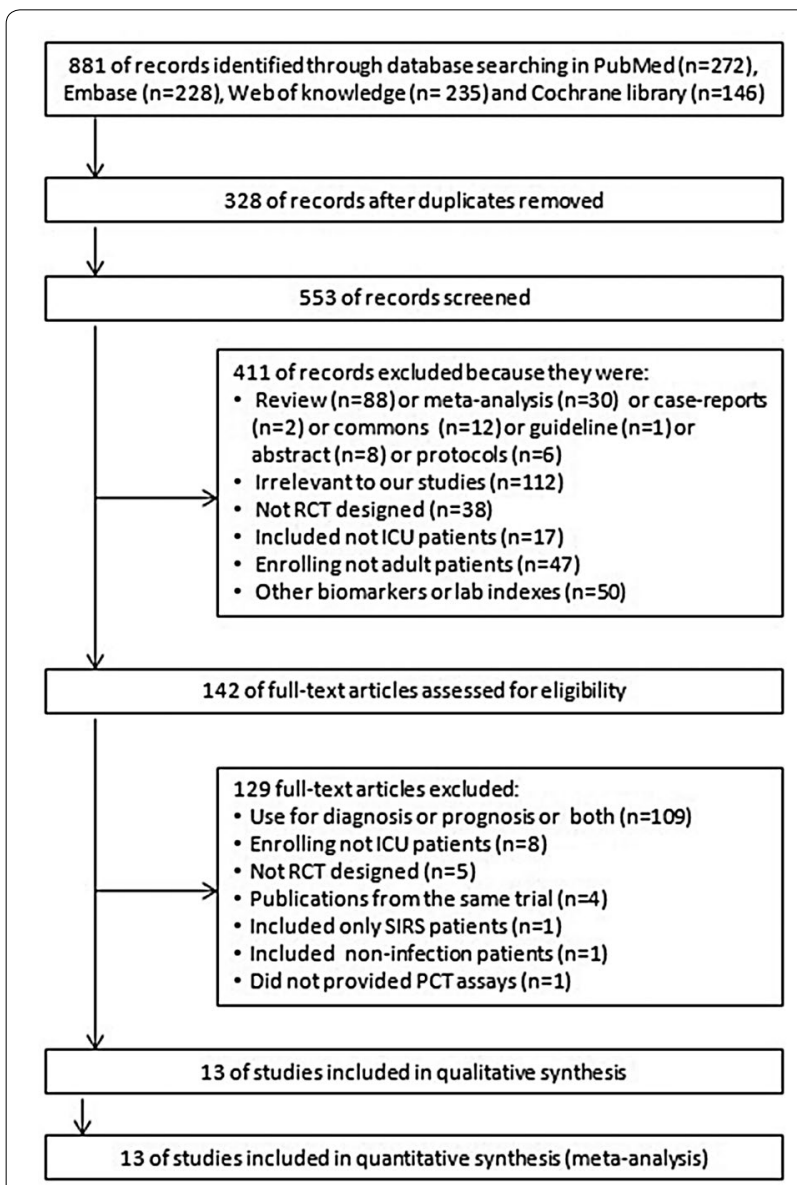

Fig. 1 Selection process for RCTs included in the meta-analysis the included RCTs (Additional file 4: Table S4). Of these included studies, seven were multicenter studies. A total of 5136 patients comprised 2588 in the PCT-guided group and 2548 in the standard care group. These studies evaluated the effects of PCT-guided strategies in antibiotic discontinuation $(n=8)[23,24,31-34,37,39]$, antibiotic initiation $(n=3)[13,30,36]$, or the combination of the antibiotic initiation and discontinuation $(n=2)[35$, 38]. Study population included surgical patients $(n=3)$ $[30,31,33]$ and mixed medical-surgical patients $(n=10)$ [13, 23, 24, 32, 34-39]. PCT assays adopted varied across the included studies.

\section{Data synthesis}

\section{Procalcitonin-guided discontinuation of antibiotics}

The use of a PCT algorithm compared with standard care to guide antibiotic discontinuation in critically ill patients was evaluated in eight RCTs [23, 24, 31-34, 37, 39]. All eight studies reported outcomes including total days with antibiotics or antibiotic-free days. The aggregated data suggested that the duration of antibiotic treatment was 1.67 days shorter in PCT-guided group $(n=3404$; MD -1.66 days; $95 \% \mathrm{CI}-2.36$ to $-0.96 ; I^{2}=71 \%$; $P<0.00001$ ) [23, 24, 31-34, 37, 39] (Fig. 2), while antibiotic-free days were 2.26 days longer $(n=2120$; MD 2.26 days; $95 \%$ CI $1.40-3.12 ; I^{2}=0 \% ; P<0.00001$ ) [23, $24,32,34]$ when compared with that of standard care group. Results showed patients in PCT-guided group had lower short-term mortality than standard care group $\left(n=3414 ;\right.$ RR $0.86 ; 95 \%$ CI $\left.0.76-0.98 ; I^{2}=0 \% ; P=0.02\right)$ [23, 24, 31-34, 37, 39] (Fig. 3), while no differences were found in ICU LOS ( $n=3326$; MD -0.00 days; $95 \% \mathrm{CI}$ -0.58 to $\left.0.58 ; I^{2}=0 \% ; P=0.99\right)[23,24,31-33,37$, 39] and hospital LOS ( $n=3290$; MD 0.43 days; $95 \% \mathrm{CI}$ -0.83 to $\left.1.70 ; I^{2}=30.4 \% ; P=0.50\right)[23,24,32,34,37$, 39]. There was significant heterogeneity in the outcome of duration of antibiotic treatment between the pooled studies. Therefore, we conducted sensitivity analyses to explore potential sources of heterogeneity. Exclusion of the trial by Bloos and colleagues resolved the heterogeneity without alerting the result $(n=2338$; MD -1.97 days; $95 \% \mathrm{CI}-2.27$ to $\left.-1.68 ; I^{2}=0 \% ; P<0.00001\right)[24,31-$ $34,37,39]$.

\section{Procalcitonin-guided initiation of antibiotics}

Three studies examined the efficacy of PCT-guided initiation of antibiotics [13, 30,36]. Only one study examined the efficacy of PCT-guided initiation of antibiotics. In this case, antibiotic consumption was comparable between groups with the treatment days represented $62.6 \%$ and $57.7 \%$ of ICU stays in the PCT and standard care groups, respectively $(P=0.11)$ [36]. There was no statistically significant difference between groups in the 


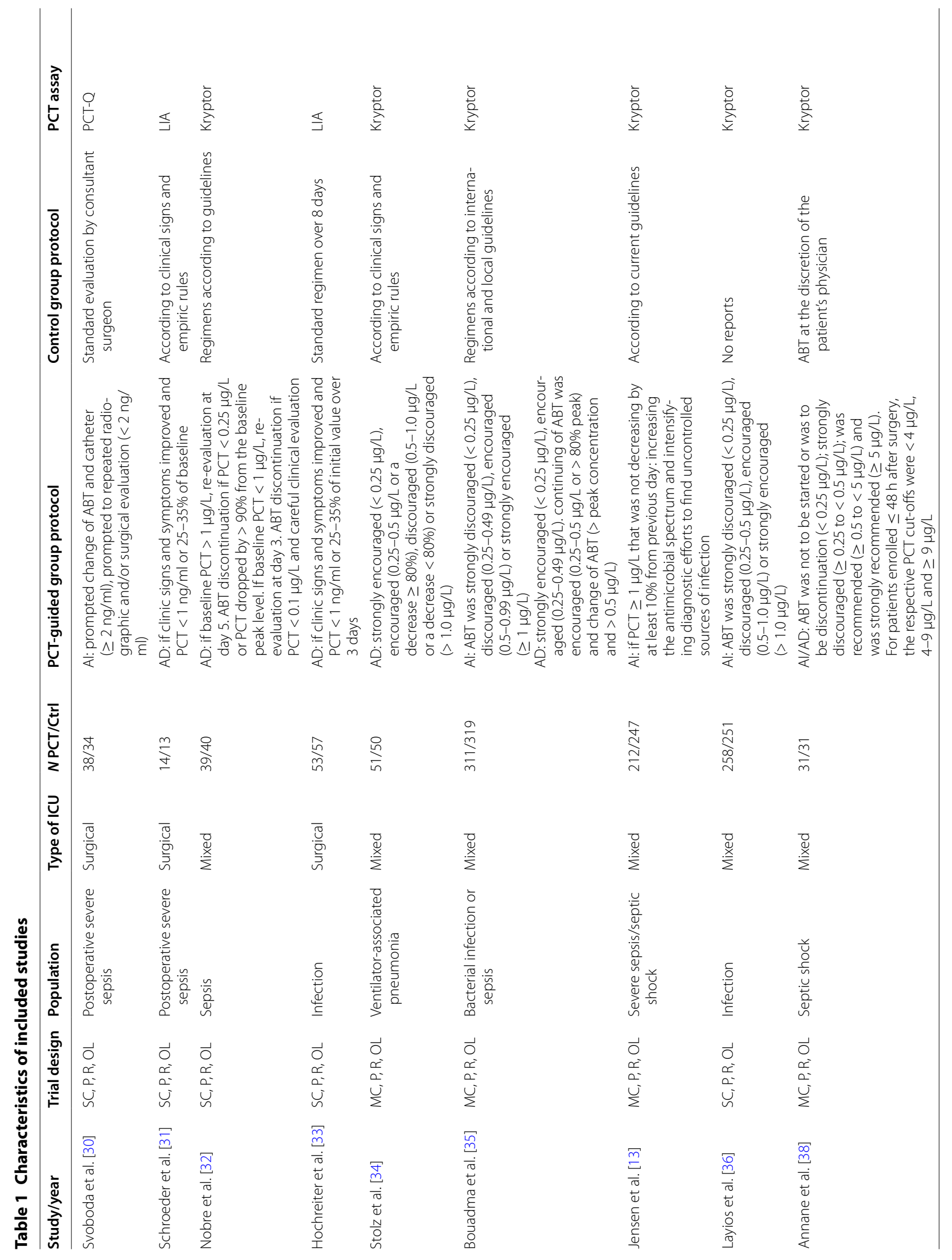




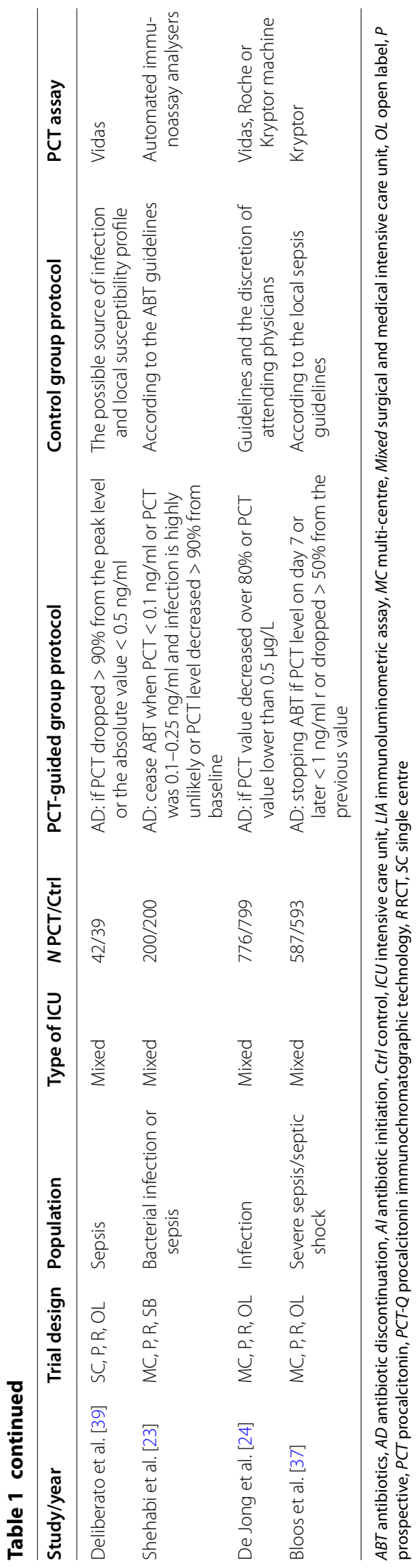




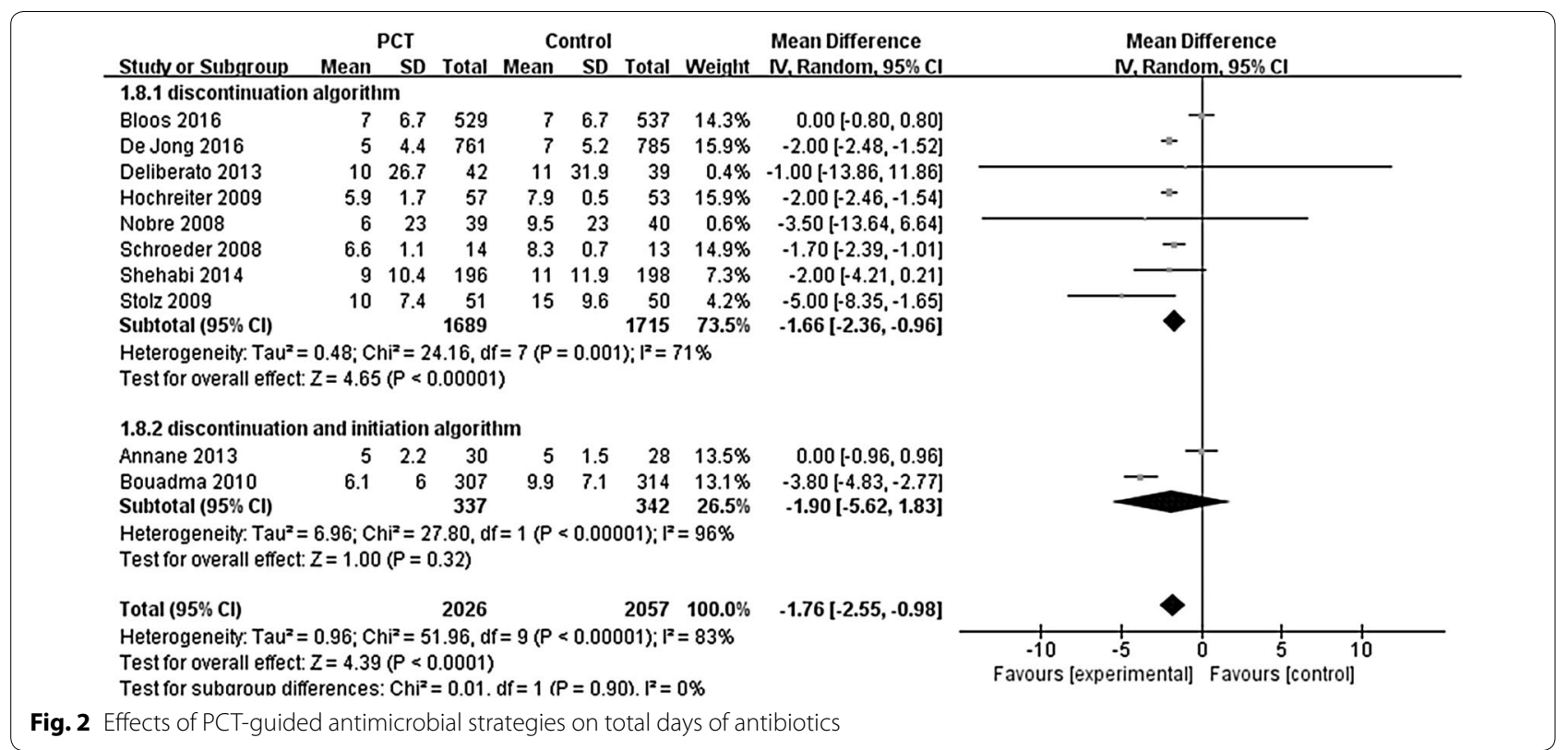

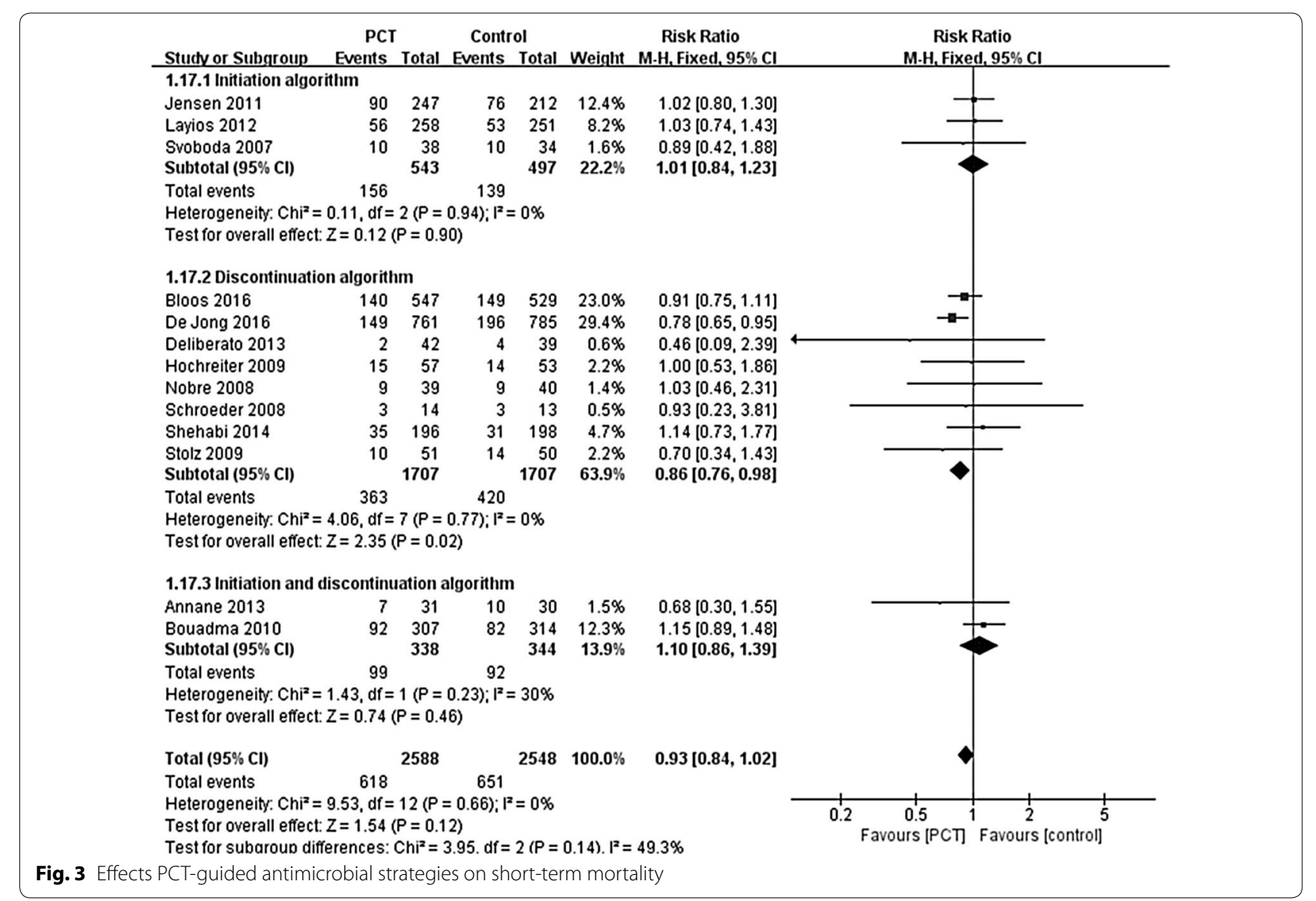


risk of short-term mortality $(n=1040$; RR 1.01; 95\% CI 0.84-1.23; $I^{2}=0 \% ; P=0.90$ ) $[13,30,36]$ (Fig. 3) or ICU LOS $(n=581 ; \mathrm{MD}-1.22$ days; $95 \% \mathrm{CI}-4.34$ to 1.90 ; $\left.I^{2}=60 \% ; P=0.44\right)[30,36]$.

\section{Procalcitonin-guided antibiotic initiation and discontinuation}

Two studies employed a PCT-guided strategy of antibiotic initiation and discontinuation [35, 38]. No differences were observed between the PCT and standard care group in total days with antibiotics $(n=679$; MD -1.90 days, 95\% CI -5.62 to $1.83 ; I^{2}=96 \% ; P=0.32$ ) (Fig. 2), antibiotic-free days ( $n=679$; MD 1.31 days; $95 \% \mathrm{CI}-1.34$ to $\left.3.95 ; I^{2}=90 \% ; P=0.33\right)$, short-term mortality $(n=682$; RR 1.10; 95\% CI 0.86-1.39; $I^{2}=30 \%$; $P=0.46$ ) (Fig. 3), the ICU LOS ( $n=682$; MD -1.45 days; $95 \% \mathrm{CI}-0.91$ to $\left.3.80 ; I^{2}=0 \% ; P=0.23\right)$, and hospital LOS $(n=679 ; \mathrm{MD}$ -0.43 days; $95 \% \mathrm{CI}-3.36$ to $2.49 ; I^{2}=0 \% ; P=0.77$ ) $[35,38]$.

Summary of findings for the effect of PCT-guided strategy antibiotic on predefined outcomes in ICU patients is described in Table 2. We did not assess the publication bias because of the limited number of studies included in each analysis.

\section{Discussion}

PCT-guided strategies had been examined in multiple studies to optimize antibiotic treatment, with conflicting results. The current meta-analysis justified a low PCT level to discontinue antibiotic treatment, which would result in a shorter duration of antibiotic treatment of about 1.67 days, as well as lower short-term mortality compared with standard care. Due to the insufficient evidence, a baseline PCT value should not be used as a marker to guide antibiotic initiation.

Our study had several strengths. The current metaanalysis provided robust evidence to support and expand the weak suggestion in the 2016 SSC guidelines, i.e. use of low PCT level to assist the clinician in the discontinuation of empiric antibiotic [12]. In addition to those RCTs in previous meta-analyses, we had included three additional RCTs recently published, and this added to the statistical power by having 3414 cases to evaluate the primary outcome. Moreover, we had stratified enrolled RCTs according to different PCT-guided strategies, in order to eliminate the potential confounding factors caused by different strategies. Though significant heterogeneity was observed among these studies, our sensitivity analyses demonstrated that the heterogeneity was resulted from the trial by Bloss et al. [23]. This study was different from the other trials in some aspects. On the one hand, it was designed as a $2 \times 2$ factorial trial and the interaction between the two treatment factors was unclear; on the other hand, in the trial [23], the clinicians used a $50 \%$ decrease from previous value as a stopping rule, which was lower than that of other studies. As a result, we also demonstrated a significant improvement in short-term mortality associated with PCT-guided antibiotic discontinuation. This had added robustness to findings of reduction in antibiotics usage, since studies have demonstrated

Table 2 Summary of findings for the effect of procalcitonin-guided strategy on predefined outcomes in intensive care unit patients

\begin{tabular}{|c|c|c|c|c|c|c|}
\hline PCT-guided strategy & Predefined outcome & Number of trials & $N$ & Estimated benefit with antibiotic & $I^{2}(\%)$ & $P$ value \\
\hline \multirow[t]{5}{*}{ ABT discontinuation } & Duration of antibiotic use & 8 & 3404 & -1.66 days $(-2.36,-0.96)$ & 71 & $P<0.0001$ \\
\hline & Antibiotic-free days & 4 & 2120 & 2.26 days $(1.40,3.12)$ & 0 & $P<0.0001$ \\
\hline & Short-term mortality & 8 & 3414 & $0.86(0.76,0.98)$ & 0 & 0.02 \\
\hline & Length of stay in ICU & 7 & 3326 & -0.00 days $(-0.58,0.58)$ & 0 & 0.99 \\
\hline & Length of stay in hospital & 6 & 3290 & 0.43 days $(-0.83,1.70)$ & 30 & 0.50 \\
\hline \multirow[t]{5}{*}{ ABT initiation } & Duration of antibiotic use & - & - & - & - & - \\
\hline & Antibiotic-free days & - & - & - & - & - \\
\hline & Short-term mortality & 3 & 1040 & $1.01(0.84,1.23)$ & 0 & 0.90 \\
\hline & Length of stay in ICU & 2 & 581 & -1.22 days $(-4.34,1.90)$ & 60 & 0.44 \\
\hline & Length of stay in hospital & - & - & - & - & - \\
\hline \multirow[t]{5}{*}{ ABT initiation and discontinuation } & Duration of antibiotic use & 2 & 679 & -1.90 days $(-5.62,1.83)$ & 96 & 0.32 \\
\hline & Antibiotic-free days & 2 & 679 & 1.31 days $(-1.34,3.95)$ & 90 & 0.33 \\
\hline & Short-term mortality & 2 & 682 & $1.10(0.86,1.39)$ & 30 & 0.46 \\
\hline & Length of stay in ICU & 2 & 682 & -1.45 days $(-0.91,3.80)$ & 0 & 0.23 \\
\hline & Length of stay in hospital & 2 & 750 & -0.43 days $(-3.36,2.49)$ & 0 & 0.77 \\
\hline
\end{tabular}

$A B T$ antibiotics, $P C T$ procalcitonin, $I C U$ intensive care unit 
that strategies aiming at restricting antibiotic overuse could help improve survival $[40,41]$. Our findings that short-term mortality was significantly reduced in the PCT-guided discontinuation group contrasted those of previous meta-analyses. Despite the fact that no heterogeneity was detected, the beneficial effect was clearly driven by the study results of de Jong et al. [24]. However, as the authors acknowledged, this study was a non-inferiority study; therefore, the beneficial effect of mortality in PCTguided group was unexpected, which merited cautious interpretation and further validation.

As for a PCT strategy that combined initiation and discontinuation of antibiotics, we found no beneficial effect with regard to any predefined outcomes. The reason for this failure may be that only two trials (one positive [35] and one negative [38]) investigated the combined strategies varying in objective and the methodology. On the other hand, reported non-compliance rate was high. For example, in a prospective, multicenter, open-label randomized trial (PRORATA trial) involving 630 non-surgical patients with suspected bacterial infections [35], patients in the PCT group had significantly more antibiotic-free days. However, the algorithm-guided treatment recommendation was not strictly followed in $53 \%$ of patients in the PCT group. Moreover, this trial [35] reported a higher standard deviation with regard to duration of antibiotic treatment as well as antibiotic-free days (possibly due to reported higher non-compliance rate), compared to that in the negative trial [38], which weaken its statistic weight in the meta-analysis. Interestingly, it was noteworthy that initial antibiotic prescription rate was similar in PCT and standard care groups, suggesting that the improvement in antibiotic-free days was more likely the result of antibiotic discontinuation, while it was less likely due to exclusion of potential infection.

In our study, we could not verify the efficacy of PCTbased antibiotic initiation strategy because we found only three RCT through the literature search $[13,30$, 36]. Of these trials, only the trial by Layios and coworkers reported the antibiotics exposure and concluded that PCT measuring for the initiation of antibiotics failed to decrease the antibiotic consummation [36]. The reasons for this failure may be that almost half of PCT serum samples were $>1 \mu \mathrm{g} / \mathrm{L}$, thus encouraging the antibiotic treatment, and the relative low proportion of patientdays with antibiotic treatment in the control group (57\%). Another reason could be related to the high non-compliance rate with the PCT-guided antibiotic initiation strategy described in the study. The authors reported that nearly $64 \%$ of patients in the PCT group received antibiotics regardless of a normal PCT level $(<0.5 \mu \mathrm{g} / \mathrm{L})$.

The incidence of non-compliance with the recommendations based on PCT algorithm, as reported in some, but not all RCTs, showed significant variability, ranging from 0 to $59 \%$. In most case of non-compliance, physicians were reluctant to stop antibiotics, even with a very low PCT level (Additional file 4: Table S4), possibly due to the concerns about the accuracy of single PCT value as a biomarker of infection [42, 43]. This might lead to unnecessarily prolonged exposure of antibiotics, which supported the robustness of our findings that implementation of PCT algorithm was associated with shorter duration of antibiotic treatment and longer antibioticfree days.

Our meta-analysis has some limitations. First, studies examining the PCT-guided strategies other than discontinuation of antibiotic treatment were scarce, with limited number of studies available as well as small number of enrolled patients. As such, data on these strategies were insufficient to draw solid conclusions. Second, the high exclusion rate of screened patients in the included studies (such as immunosuppressed patients and those requiring long-term antibiotic therapy) precluded generalization of the study results. Third, antibiotic strategy in the control group (indications to initiate and discontinue antibiotics) was not specified in most studies. Whether the variation in antibiotic strategy, if any, in the control group might have affected the results of our meta-analysis is unclear. Thus, a more uniform approach to evaluating and reporting standard care related to antibiotic use would be needed in future studies. Fourth, the uneven distribution of different underlying diseases among included studies might also exert a prognostic value. Of note, in the two recently published systematic reviews and individual patient data metaanalysis [44, 45], Schuetz et al. demonstrated with sufficient evidence that PCT-guided antibiotic treatment in patients with acute respiratory infections reduced antibiotic exposure and side effects and improved survival. Finally, different cut-off values of PCT and different PCT measurements were reported across included studies, which might also lead to bias in our results. We had originally tried to perform subgroup analyses exploring studies according to all the diversities. However, there were insufficient data.

\section{Conclusions}

In summary, based on the results of our meta-analysis, we recommend use of PCT to guide antibiotic discontinuation, which was associated with a reduction in antibiotic exposure and lower short-term mortality. Further studies are needed to define the optimal cut-off value of PCT for antibiotic discontinuation and to generalize our findings in other patient population including immunocompromised patients and those received long-term antibiotic therapy in ICU. 


\section{Additional files}

Additional file 1: Table S1. Summary of the RCTs included by previous and current meta-analysis.

Additional file 2: Table S2. Predefined outcomes of the included RCTs. Additional file 3: Table S3. Risk of bias table for included randomized control trials.

Additional file 4: Table S4. Non-compliance rate reported in the included RCTs.

\section{Abbreviations}

Cl: confidence interval; ICU: intensive care unit; IQR: interquartile range; LOS: length of stay; MD: mean difference; PCT: procalcitonin; RR: risk ratio; RCTs: randomized controlled trials; SD: standard deviations; VAP: ventilator-associated pneumonia.

\section{Authors' contributions}

$\mathrm{H}$-BH contributed to the conception of the study, data collection, analysis, and drafting of the article. WJ and C-YW contributed to data collection and analysis. LW and J-MP contributed to design and revisions of this manuscript. $\mathrm{BD}$ was responsible for the integrity of the work as a whole, from inception to publication of the article. All authors read and approved the final manuscript.

\section{Author details}

${ }^{1}$ Medical ICU, Peking Union Medical College Hospital, Peking Union Medical College and Chinese Academy of Medical Sciences, 1 Shuai Fu Yuan, Beijing 100730, People's Republic of China. ${ }^{2}$ Department of Critical Care Medicine, The First Affiliated Hospital of Fujian Medical University, Fuzhou, China.

\section{Acknowledgements}

We thank Jing-Chao Luo, MD, for his assistance in searching the literature.

\section{Competing interests}

The authors declare that they have no competing interests.

\section{Availability of data and materials}

All data generated or analysed during this study are included in this published article.

\section{Consent for publication}

Not applicable.

\section{Ethics approval and consent to participate}

Not applicable.

\section{Funding}

Cams Innovation Fund for Medical Sciences (2016-12M-1-014).

\section{Publisher's Note}

Springer Nature remains neutral with regard to jurisdictional claims in published maps and institutional affiliations.

Received: 7 July 2017 Accepted: 12 November 2017

Published online: 22 November 2017

\section{References}

1. Braykov NP, Morgan DJ, Schweizer ML, et al. Assessment of empirical antibiotic therapy optimisation in six hospitals: an observational cohort study. Lancet Infect Dis. 2014;14(14):1220-7.

2. Llor C, Bjerrum L. Antimicrobial resistance: risk associated with antibiotic overuse and initiatives to reduce the problem. Ther Adv Drug Saf. 2014;5(6):229-41.
3. Dellit TH, Owens RC, McGowan JE Jr, et al. Infectious Diseases Society of America and the Society for Healthcare Epidemiology of America guidelines for developing an institutional program to enhance antimicrobial stewardship. Clin Infect Dis. 2007;44(4):263-4.

4. Dimopoulos G, Poulakou G, Pneumatikos IA, Armaganidis A, Kollef MH, Matthaiou DK. Short- vs long-duration antibiotic regimens for ventilatorassociated pneumonia: a systematic review and meta-analysis. Chest. 2013;144(6):1759-67.

5. Najafi A, Khodadadian A, Sanatkar M, et al. The comparison of procalcitonin guidance administer antibiotics with empiric antibiotic therapy in critically ill patients admitted in intensive care unit. Acta Medica Iranica. 2015;53(9):562-7.

6. Evans HL, Lefrak SN, Lyman J, et al. Cost of gram-negative resistance. Crit Care Med. 2007;35(1):89-95.

7. Vincent JL, Rello J, Marshall J, et al. International study of the prevalence and outcomes of infection in intensive care units. J Am Med Assoc. 2009;302(21):2323-9.

8. Luyt CE, Combes A, Reynaud C, et al. Usefulness of procalcitonin for the diagnosis of ventilator-associated pneumonia. Intensive Care Med. 2008;34(8):1434-40.

9. Simon L, Lacroix J. Serum procalcitonin and C-reactive protein levels as markers of bacterial infection: a systematic review and meta-analysis. Clin Infect Dis. 2004;39:206-17.

10. Wacker C, Prkno A, Brunkhorst FM, Schlattmann P. Procalcitonin as a diagnostic marker for sepsis: a systematic review and meta-analysis. Lancet Infect Dis. 2013;13(5):426-35.

11. Schuetz P, Muller B, Christ-Crain M, et al. Procalcitonin to initiate or discontinue antibiotics in acute respiratory tract infections. Evid Based Child Health. 2013:8:1297-371.

12. Rhodes A, Evans LE, Alhazzani W, et al. Surviving sepsis campaign: international guidelines for management of sepsis and septic shock. Crit Care Med. 2017;45(3):486-552.

13. Jensen $J U$, Hein $L$, Lundgren $B$, et al. Procalcitonin-guided interventions against infections to increase early appropriate antibiotics and improve survival in the intensive care unit: a randomized trial. Crit Care Med. 2011;39(9):2048-58.

14. Kopterides P, Siempos II, Tsangaris I, Tsantes A, Armaganidis A. Procalcitonin-guided algorithms of antibiotic therapy in the intensive care unit: a systematic review and meta-analysis of randomized controlled trials. Crit Care Med. 2010;38(11):2229-41.

15. Heyland DK, Johnson AP, Reynolds SC, Muscedere J. Procalcitonin for reduced antibiotic exposure in the critical care setting: a systematic review and an economic evaluation. Crit Care Med. 2011;39(7):1792-9.

16. Agarwal R, Schwartz DN. Procalcitonin to guide duration of antimicrobial therapy in intensive care units: a systematic review. Clin Infect Dis. 2011;53(4):379-87.

17. Schuetz P, Chiappa V, Briel M, Greenwald JL. Procalcitonin algorithms for antibiotic therapy decisions: a systematic review of randomized controlled trials and recommendations for clinical algorithms. Arch Intern Med. 2011;171(15):1322-31.

18. Soni NJ, Pitrak DL, Aronson N, Samson DJ, Galaydick JL, Vats V. Procalcitonin-guided antibiotic therapy. Agency for Healthcare Research and Quality. 2012

19. Matthaiou DK, Ntani G, Kontogiorgi M, Poulakou G, Armaganidis A, Dimopoulos G. An ESICM systematic review and meta-analysis of procalcitonin-guided antibiotic therapy algorithms in adult critically ill patients. Intensive Care Med. 2012;38(6):940-9.

20. Tang H, Huang $T$, Jing J, Shen $H$, Cui W. Effect of procalcitonin-guided treatment in patients with infections: a systematic review and metaanalysis. Infection. 2009;37(6):497-507.

21. Prkno A, Wacker C, Brunkhorst FM, Schlattmann P. Procalcitonin-guided therapy in intensive care unit patients with severe sepsis and septic shock - a systematic review and meta-analysis. Crit Care. 2013;17(6):R291.

22. Westwood M, Ramaekers B, Whiting P, et al. Procalcitonin testing to guide antibiotic therapy for the treatment of sepsis in intensive care settings and for suspected bacterial infection in emergency department settings: a systematic review and cost-effectiveness analysis. Health Technol Assess. 2015;19(96):1-236.

23. Bloos F, Trips E, Nierhaus A, et al. Effect of sodium selenite administration and procalcitonin-guided therapy on mortality in patients with severe 
sepsis or septic shock: a randomized clinical trial. JAMA Intern Med. 2016;176(9):1266-76.

24. de Jong E, van Oers JA, Beishuizen A, et al. Efficacy and safety of procalcitonin guidance in reducing the duration of antibiotic treatment in critically ill patients: a randomised, controlled, open-label trial. Lancet Infect Dis. 2016;16(7):819-27.

25. Moher D, Liberati A, Tetzlaff J, Altman DG, Group TP. Preferred reporting items for systematic reviews and meta-analyses: the PRISMA statement. J Chin Integr Med. 2010;8(5):336-41.

26. Huang H, Li Y, Ariani F, Chen X, Lin J. Timing of tracheostomy in critically ill patients: a meta-analysis. PLoS ONE. 2014;9(3):e92981.

27. Wang $F, W u Y, B o L$, et al. The timing of tracheotomy in critically ill patients undergoing mechanical ventilation: a systematic review and meta-analysis of randomized controlled trials. Chest. 2011;140(140):1456-65.

28. Higgins JP, Altman DG, Gotzsche PC, et al. The Cochrane Collaboration's tool for assessing risk of bias in randomised trials. BMJ. 2011;343(oct18 2):d5928.

29. Wan X, Wang W, Liu J, Tong T. Estimating the sample mean and standard deviation from the sample size, median, range and/or interquartile range. BMC Med Res Methodol. 2014;14(8):735-46.

30. Svoboda P, Kantorová I, Scheer P, Radvanova J, Radvan M. Can procalcitonin help us in timing of re-intervention in septic patients after multiple trauma or major surgery? Hepatogastroenterology. 2007;54(74):359-63.

31. Schroeder $\mathrm{S}$, Hochreiter $\mathrm{M}$, Koehler $\mathrm{T}$, et al. Procalcitonin (PCT)-guided algorithm reduces length of antibiotic treatment in surgical intensive care patients with severe sepsis: results of a prospective randomized study. Langenbecks Arch Surg. 2009;394(2):221-6.

32. Nobre V, Harbarth S, Graf JD, Rohner P, Pugin J. Use of procalcitonin to shorten antibiotic treatment duration in septic patients: a randomized trial. Am J Respir Crit Care Med. 2008;177(5):498-505.

33. Hochreiter M, Kohler T, Schweiger AM, et al. Procalcitonin to guide duration of antibiotic therapy in intensive care patients: a randomized prospective controlled trial. Crit Care. 2009;13(3):R83.

34. Stolz D, Smyrnios N, Eggimann P, et al. Procalcitonin for reduced antibiotic exposure in ventilator-associated pneumonia: a randomised study. Eur Respir J. 2009;34(6):1364-75.
35. Bouadma L, Luyt CE, et al. Use of procalcitonin to reduce patients' exposure to antibiotics in intensive care units (PRORATA trial): a multicentre randomised controlled trial. Lancet. 2010;375(9713):463-74.

36. Layios N, Lambermont B, Canivet JL, et al. Procalcitonin usefulness for the initiation of antibiotic treatment in intensive care unit patients. Crit Care Med. 2012;40(8):2304-9.

37. Shehabi Y, Sterba M, Garrett PM, et al. Procalcitonin algorithm in critically ill adults with undifferentiated infection or suspected sepsis. A randomized controlled trial. Am J Respir Crit Care Med. 2014;190(10):1102-10.

38. Annane D, Maxime V, Faller JP, et al. Procalcitonin levels to guide antibiotic therapy in adults with non-microbiologically proven apparent severe sepsis: a randomised controlled trial. BMJ Open. 2013;3(2):e002186.

39. Deliberato RO, Marra AR, Sanches PR, et al. Clinical and economic impact of procalcitonin to shorten antimicrobial therapy in septic patients with proven bacterial infection in an intensive care setting. Diagn Microbiol Infect Dis. 2013;76(3):266-71.

40. Levy SB, Marshall B. Antibacterial resistance worldwide: causes, challenges and responses. Nat Med. 2004;10(12 suppl):S122-9.

41. Cohen ML. Epidemiology of drug resistance: implications for a postantimicrobial era. Science. 1992;257(257):1050-5.

42. Schuetz $P$, Affolter $B$, Hunziker $S$, et al. Serum procalcitonin, C-reactive protein and white blood cell levels following hypothermia after cardiac arrest: a retrospective cohort study. Eur J Clin Invest. 2010;40(4):376-81.

43. Jung B, Molinari N, Nasri M, et al. Procalcitonin biomarker kinetics fails to predict treatment response in perioperative abdominal infection with septic shock. Crit Care. 2013;17(5):R25.

44. Schuetz P, Wirz Y, Sager R, et al. Procalcitonin to initiate or discontinue antibiotics in acute respiratory tract infections. Cochrane Database Syst Rev. 2017;10:CD007498.

45. Schuetz P, Wirz Y, Sager R et al. Effect of procalcitonin-guided antibiotic treatment on mortality in acute respiratory infections: a patient level meta-analysis. Lancet Infect Dis. 2017. https://doi.org/10.1016/ S1473-3099(17)30592-3.

\section{Submit your manuscript to a SpringerOpen ${ }^{\odot}$ journal and benefit from:}

- Convenient online submission

- Rigorous peer review

- Open access: articles freely available online

- High visibility within the field

- Retaining the copyright to your article

Submit your next manuscript at $\boldsymbol{\nabla}$ springeropen.com 